\title{
El aprendizaje de los objetivos de desarrollo sostenible en las asignaturas de microeconomía y macroeconomía
}

\author{
Luisa Marti ${ }^{\mathrm{a}}$, Rosa Puertas ${ }^{\mathrm{b}}$, Consuelo Calafat ${ }^{\mathrm{c}}$ \\ ${ }^{a}$ Universitat Politecnica de Valencia, Valencia, España, mlmarti@esp.upv.es, ${ }^{\text {UUniversitat Politecnica }}$ \\ de Valencia, Valencia, España, rpuertas@esp.upv.es, 'Universitat Politecnica de Valencia, Valencia, \\ España, chelo@esp.upv.es
}

\section{Resumen}

Tras cinco años (septiembre 2015) desde que se estableció el acuerdo de Naciones Unidas "Transformar nuestro mundo: la Agenda 2030 para el Desarrollo Sostenible", la universidad se ha convertido en un canal idóneo para transmitir el conocimiento de los Objetivos de Desarrollo Sostenible (ODS), bajo el lema de "no dejar a nadie atrás" proponen erradicar la pobreza, reducir las desigualdades y cuidar el medio ambiente. Siguiendo con las recomendaciones de la CRUE sobre la necesidad de las universidades de realizar reflexiones estratégicas en materia de integración de ODS en la política universitaria, es necesario incluir estos conceptos en las materias del grado de Administración y Dirección de Empresas (ADE). En este contexto, las asignaturas de microeconomía y macroeconomía, ambas pertenecientes al área de Economía Aplicada del grado de ADE, resultan idóneas para instruir a sus estudiantes en determinados ODS. El objetivo del artículo es introducir innovación docente relacionando algunos ODS con los temas de los programas de microeconomía y macroeconomía. Teniendo en cuenta que microeconomía es una materia centrada en el comportamiento del consumidor, el productor y el Estado, está relacionada con los ODS: (7) "Energía asequible y no contaminante", (9) "Industria, innovación e infraestructuras" y (12) "Producción y consumo responsable". Mientras que la asignatura de macroeconomía tiene una visión más global del país, donde se analizan políticas económicas vinculadas con los siguientes ODS: (8) "Trabajo decente y crecimiento económico", (10) "Reducción de las desigualdades" y (12) "Producción y consumo responsable". El resultado del aprendizaje consistirá en conseguir que los graduados en ADE adquieran conocimientos sobre determinados ODS y tengan las capacidades necesarias para implementarlos en la empresa donde trabajen en un futuro. 
El aprendizaje de los objetivos de desarrollo sostenible en las asignaturas de microeconomía y macroeconomía

Palabras clave: Objetivos de Desarrollo Sostenible, Microeconomia, Macroeconomia.

\section{Introducción}

La Conferencia de Rectores de las Universidades Españolas (CRUE) está viendo la necesidad de iniciar un proceso de reflexión estratégica sobre la integración de los Objetivos de Desarrollo Sostenible (ODS) en el contexto de la educación superior, lo que implica abordar estos objetivos de forma transversal en las políticas universitarias, así como integrarlos en los distintos ámbitos de acción de la universidad: la formación, la investigación y la extensión universitaria (CRUE, 2019a). Los centros educativos, debido a su labor de generación y difusión del conocimiento y su preeminente situación dentro de la sociedad, están llamados a desempeñar un papel fundamental en el logro de los ODS. Según Remacha (2017) el principal destinatario de los ODS son los países, que tienen la responsabilidad de desarrollar políticas, planes y programas de desarrollo sostenible y de llevar un seguimiento y un control de los progresos conseguidos, y por tanto su análisis tiene una perspectiva macroeconómica. Sin embargo, los ODS reconocen explícitamente el rol fundamental que las empresas pueden y deben desempeñar en su logro, suponiendo en tal caso que el análisis microeconómico también es importante para su conocimiento. En este contexto, el objetivo del artículo es establecer una propuesta de aprendizaje para introducir los ODS en los programas de microeconomía y macroeconomía del grado de Administración y Dirección de Empresas (ADE).

Teniendo en cuenta que la microeconomía es una materia centrada en el comportamiento del consumidor, el productor y el Estado, es posible relacionarla con los siguientes ODS: (7) "Energía asequible y no contaminante", (9) "Industria, innovación e infraestructuras" y (12) "Producción y consumo responsable". Mientras que la asignatura de macroeconomía tiene una visión más global del país, donde se analizan políticas económicas vinculadas con los siguientes ODS: (8) "Trabajo decente y crecimiento económico", (10) "Reducción de las desigualdades" y (12) "Producción y consumo responsable". El resultado del aprendizaje consistirá en conseguir que los graduados en ADE adquieran conocimientos sobre determinados ODS y tengan las capacidades necesarias para implementarlos en la empresa donde trabajen en un futuro.

El resto del artículo se divide de la siguiente forma. En la sección 2 se explican los ODS en el contexto general de los estudios de educación superior. En la sección 3 se detalla que relación existen entre algunos objetivos y la materia de microeconomía. En la sección 4 se hace referencia a un análisis macroeconómico bajo la perspectiva de algunos ODS 
relacionados con el programa de la asignatura. Por último, en la sección 5 se resumen las conclusiones.

\section{Relación de los ODS con los estudios de educacion superior}

Tras cinco años (septiembre 2015) desde el acuerdo de Naciones Unidas "Transformar nuestro mundo: la Agenda 2030 para el Desarrollo Sostenible", la universidad se ha convertido en un canal idóneo para transmitir los ODS, bajo el lema de "no dejar a nadie atrás" proponen erradicar la pobreza, reducir las desigualdades y cuidar el medio ambiente (PNUD, 2016). Según las recomendaciones de la CRUE sobre la necesidad de las universidades de realizar reflexiones estratégicas en materia de integración de ODS en la política universitaria, es necesario introducir estos conceptos en las materias del grado de ADE. En concreto, a propuesta de los autores se distinguen tres pasos del aprendizaje:

1. Entender los ODS: De forma que el alumno se familiarizará con los ODS, lo que implica conocer en qué consisten cada uno de los objetivos y cuáles son sus metas correspondientes. Así, comprenderá las oportunidades y las responsabilidades que individual y colectivamente representan para las empresas y los países.

2. Priorizar: Partiendo de la base de que no todos los ODS serán igualmente relevantes para las distintas asignaturas y de que éstas no tendrán la misma capacidad de influencia sobre ellos, cada docente deberá evaluar su impacto para definir las áreas de actuación.

3. Diseñar estrategias de innovación docente: Según el programa de cada asignatura el docente deberá establecer unas actividades que permitan profundizar en el conocimiento de los ODS, relacionando dichos objetivos con la materia aprendida.

Las asignaturas objeto de análisis se imparten en $1^{\circ}$ y $2^{\circ}$ del grado de ADE, siendo de formación básica microeconomía I y macroeconomía I, y de formación obligatoria microeconomía II y macroeconomía II. Cada una de ellas tiene una carga docence de 6 créditos ECTS dividos en teoría de aula, y prácticas de aula y laboratorio. Como se indica en los objetivos de esta investigación se desarrollarán estos pasos en las asignaturas de microeconomía y macroeconomía, dado que son asignaturas troncales que facilitan la comprensión del proceso de asignación de recursos destinadas a futuros profesiones que pueden desempeñar funciones de responsabilidad en las administraciones públicas o empresas privadas. 


\section{Relación entre la materia de microeconomía y los ODS}

La microeconomía estudia el comportamiento de los compradores o demandantes, junto con el de los productores o empresas y el Estado. Todos estos agentes verán modificadas sus estrategias de conducta con la incorporación de los ODS en el funcionamiento. En primer lugar, el alumno deberá conocer los 17 ODS incluidos en la Agenda 2030, para que tome conciencia de la importancia del desarrollo sostenible global, como vía para garantizar la armonización de la sociedad en todas sus facetas. En segundo lugar, se identificaran aquellos objetivos más relacionados con la materia impartida en las asignaturas de microeconomía. Concretamente se proponen los siguientes: ODS (7): "Energía asequible y no contaminantes": según Remacha (2017) la inversión en fuentes de energía limpia, como la solar, eólica y termal, es un asunto prioritario para el sector privado de cara a poder garantizar el acceso universal a electricidad asequible para 2030. Por tanto, habrá nuevas inversiones que la empresa debe financiar pare percibir la mejora en su funcionamiento. ODS (9) "Industria, Innovación e Infraestructura", concretamente siguiendo a Medina (2019) empresas como Inditex, Nestlé, Unilever o Mercadona han comprendido la importancia de los ODS y por eso han alineado su estrategia con la Agenda 2030 para el Desarrollo Sostenible de Naciones Unidas, adoptando un firme compromiso con el cumplimiento de los objetivos, basada en acciones como la optimización de la logística, ahorro y eficiencia energética y gestión integral de residuos. ODS (12): "Producción y Consumo responsable": Este objetivo está relacionado con los demandantes, ya que la inclusión de los ODS en la empresa puede considerarse como un medio para atraer a nuevos tipos de consumidores, los cuales están cada vez más preocupados por la cuestión ambiental y social y buscan productos sostenibles (amigables ecológicamente, libres de crueldad animal o sin esclavitud ni trabajo infantil).

Por último, se diseñarán estrategias para incorporar los ODS en las actividades de aprendizaje programadas, dado que los ODS son una realidad que evoluciona de forma continua con interacción con los agentes sociales y económico. La forma de tratarlos será en las clases prácticas a partir de estadísticas oficiales, pudiendo realizar un seguimiento continuo de los avances conseguidos por los paises. Frente al formato tradicional donde los estudiantes realizan un trabajo sobre las características de un sector concreto utilizando los conceptos teóricos aprendidos en el aula, se propone como alternativa que relacionen aspectos concretos del tema analizado con los ODS aprendidos en las clases practicas (Tabla 1).

El desarrollo del trabajo sobre un sector económico siguiendo el contenido tradicional era elaborado a partir de la información económica-financiera que se obtiene de las empresas 
que figuran en la base de datos SABI (Sistema de Análisis de Balances Ibéricos). Pero es importante que vean como empresas importantes ponen en práctica el cumplimiento de los ODS, para ello profundizarán en los informes más actualizados ${ }^{1}$ de empresas grandes y concienciadas con la Agenda 2030, para obtener la información requerida.

Tabla 1. Comparativa de contenidos en microeconomía

\begin{tabular}{|c|c|}
\hline Contenido Tradicional & Contenido con ODS \\
\hline $\begin{array}{l}\text { Identificar los costes en materias primas de las } \\
\text { principales empresas del sector económico }\end{array}$ & $\begin{array}{l}\text { Identificar si las empresas se preocupan por } \\
\text { cumplir el ODS } 7 \text { utilizando energias } \\
\text { asequibles y no contaminates }\end{array}$ \\
\hline $\begin{array}{l}\text { Analizar el tipo de industria al que pertenecen } \\
\text { las principales empresas del sector económico }\end{array}$ & $\begin{array}{l}\text { Analizar si las empresas del sector son capaces } \\
\text { de cumplir con el ODS } 9 \text { sobre industria, } \\
\text { innovacion e infraestructuras }\end{array}$ \\
\hline $\begin{array}{l}\text { Cuantificar las ventas de las principales } \\
\text { empresas del sector económico y el tipo de } \\
\text { clientes que tienen }\end{array}$ & $\begin{array}{l}\text { Estudiar si tanto la producción de las empresas } \\
\text { del sector como el consumo de sus clientes es } \\
\text { responsable, para identificar el grado de } \\
\text { cumplimiento del ODS } 12\end{array}$ \\
\hline $\begin{array}{l}\text { Proponer estrategias de mejora en sus } \\
\text { beneficios }\end{array}$ & $\begin{array}{l}\text { Señalar las ventajas e inconvenientes de las } \\
\text { actuaciones innovadoras para cumplir los ODS }\end{array}$ \\
\hline
\end{tabular}

Fuente: Elaboración propia

En referencia al primer punto del contenido con ODS, el cumplimiento del objetivo 7 supone reducir costes y utilizar energía limpia abriendo grandes oportunidades hacia la expansión de las infraestructuras y el desarrollo de tecnologías bajas en carbono. Por tanto, su identificación es importante para el futuro empresarial. El segundo punto, en relación con el objetivo 9, para muchas otras empresas, este objetivo puede ser un incentivo para servirse de la investigación y el desarrollo (I+D) en la mejora de los procesos y como vía para fomentar el valor añadido de sus productos y servicios. Los avances tecnológicos podrán reducir costes o convertirse en una nueva fuente de ingresos. El tercer punto, hace referencia al objetivo 12, implicando que los modelos de negocio tenderán hacia el concepto de «economía circular», un sistema mediante el cual el valor de los productos, los materiales y los recursos se mantenga en la economía durante el mayor tiempo posible y que permita reducir al mínimo la generación de residuos. Respecto al último punto sobre ventajas e inconvenientes de las actuaciones innovadoras para cumplir los ODS, hay que tener en cuenta que la consecución de los ODS creará el escenario idóneo para que el sector privado pueda desarrollar su actividad: mercados estables, regulados y competitivos, sistemas financieros transparentes e instituciones sin corrupción y bien gobernadas,

\footnotetext{
${ }^{1}$ También es conveniente que el alumno analice informes generales como RED ESPAÑOLA DEL PACTO MUNDIAL (2020) o SDSN (2017).
} 
El aprendizaje de los objetivos de desarrollo sostenible en las asignaturas de microeconomía y macroeconomía

materias primas y energía accesibles, consumidores con poder adquisitivo y empleados cualificados (Remacha, 2017). Y por tanto, habrá más ventajas que inconvenientes.

\section{Análisis macroeconómico bajo la óptica de algunos ODS}

La macroeconomía estudia el comportamiento de la economía en su conjunto, definiendo las variables agregadas que la caracterizan: nivel global de producción, empleo y precios de un determinado territorio. Las decisiones de los agentes económicos se analizan de forma conjunta: el total de consumidores; todas las empresas de la economía, sin distinguir sectores económicos; el agregado de instituciones públicas; y el sector exterior, como la fusión de economías del resto del mundo. La evolución de las variables macroeconómicas (producción y empleo) está muy relacionada con el logros de los ODS. Respecto a la evolución del empleo mundial, el informe Better Business, Better World, elaborado por la Comisión de Comercio y Desarrollo Sostenible ya identificó en 2017 que lograr los ODS podía generar al menos 12 billones de dólares en oportunidades hasta 2030 y potencialmente el doble o el triple, además de 380 millones de empleos globales.

Siguiendo el esquema de la asignatura de macroeconomía, en primer lugar se presentarán las cinco áreas contextuales de los ODS conocidas como las 5P: personas, planeta, prosperidad, paz y alianzas, con la finalidad de familiarizar a los alumnos con los conceptos básicos y el paradigma que gira en torno a la sostenibilidad ambiental, social y económica. En segundo lugar, se discriminarán aquellos objetivos que guarden una relación más estrecha con la materia impartida en macroeconomía. Esta selección se realizará teniendo en cuenta que el gran potencial para el crecimiento sostenible de la economía, tanto a nivel global como local, puede verse afectado por aspectos como el trabajo decente (ODS 8), la reducción de las desigualddes (ODS 10) y la producción y consumo responsable (ODS 12). La accion en estas áreas desbloquearía millones de beneficios para el Producto Interior Bruto (PIB) global, creando nuevos empleos, al mismo tiempo contribuye a alcanzar las metas de la Agenda 2030. Con ello, la economía se basa en la igualdad y en modelos de producción circular, para ser mas prospera (Red Española del Pacto Mundial de Naciones Unidas, 2020).

Por último, se se diseñarán metodologías activas de aprendizaje para introducir los ODS en las actividades realizadas con los alumnos durante las prácticas de laboratorio. Actualmente, se estudia la evolución de las principales variables macroeconómicas (PIBpm, Renta Disponible, Consumo, Inversión, Gasto Público, etc.). Se propone ampliar la formación de estas variables desde el punto de vista de los logros de los ODS, para ello los alumnos realizarán y expondrán un trabajo en grupo. El objetivo será que conozcan la 
implicación de los ODS en la evolución del crecimiento económico y social tanto en España como en otros países de europeos.

El trabajo se basará en la obtención de indicadores del progreso de los ODS seleccionados desde las bases de datos de Eurostat (https:/ec.europa.eu/eurostat/web/sdi/indicators), tanto de la economía española como de otros países europeos. Se trabajará estos indicadores en las prácticas de la asignatura, desarrolladas en aulas informáticas, todas ellas tienen acceso a las bases de datos. En grupos de 3-5 alumnos se realizará un informe que será expuesto en la última práctica programada Cada grupo elegirá un país europeo y deberá obtener los indicadores de los tres ODS seleccionados, comparándolos con los correspondientes a España.

Los indicadores que se analizan en el ODS 8 están relacionados con la riqueza económica, el mercado laboral y las condiciones de trabajo a través del PIB per cápita, empleo, desempleo a largo plazo y personas fallecidas en accidentes laborales. El seguimiento del ODS 10 se centra en el progreso logrado en la reducción de las desigualdades entre y dentro de los países y en el logro de la inclusión social y la migración segura. Los principales indicadores utilizados son: comparación entre el ingreso bruto de los hogares, desiguladad en la distribución del ingresos entre los hogares (divide el ingreso del 20\% más rico de la población por el ingreso del $20 \%$ más pobre) y la participación en los ingresos del $40 \%$ inferior (habría igualdad total si el 40\% de la población tuviera el $40 \%$ del ingreso total). El ODS 12 está relacionado con la cantidad de recursos disponbiles, y recuerda que nuestro planeta tiene recursos finitos. Para enfrentar este desafío, es necesario cambiar la forma de producir, consumir y generar residuos, hacia un modelo de producción circular. Los indicadores de seguimiento son la cantidad de residuos generados, tanto en el sector privado (hogares y empresas) como en el público, el consumo total de energía y la tasa de reciclaje.

La realización de este trabajo ampliará los conocimientos de los alumnos según indica la Tabla 2, y potenciará su pensamiento crítico.

Tabla 2. Comparativa de contenidos en macroeconomía

\begin{tabular}{|lllll|}
\hline \multicolumn{3}{|c|}{ Contenido Tradicional } & \multicolumn{3}{c|}{ Contenido con ODS } \\
Analizar la evolución de crecimiento & $\begin{array}{l}\text { Analizar la riqueza económica } \\
\text { económico (PIBpm y sus componentes) }\end{array}$ & y & $\begin{array}{l}\text { cumplimiento al ODS8 en España } \\
\text { compasión con otros países europeos }\end{array}$ \\
mercado de trabajo en España & en & \\
$\begin{array}{l}\text { Compara evolución del PIBpm en términos } \\
\text { reales (tasas de variación de la producción) en }\end{array}$ & $\begin{array}{l}\text { Estudiar de la evolución de la producción y las } \\
\text { desigualdades (ODS 10) dentro del país y su } \\
\text { la economía española en los últimos 10 años }\end{array}$ & $\begin{array}{l}\text { comparación con otros países europeos } \\
\text { Descomponer las PIB según las rentas } \\
\text { generadas por los factores de producción }\end{array}$ & $\begin{array}{l}\text { Analizar la cantidad de recursos de la economía } \\
\text { y los residuos que se generan (ODS12) }\end{array}$ \\
\hline
\end{tabular}

Fuente: Elaboración propia 


\section{Conclusiones}

Actualmente, la raza humana se enfrenta a importantes objetivos sin fronteras ni precedentes, que azotan a todo el planeta. Se precisan respuestas contundentes para solucionar problemas globales, como el cambio climático, la pobreza, la precariedad educativa, las desigualdades de género, etc. En los últimos dos cursos académicos se ha comenzado a introducir los ODS en la materias analizadas, obteniendo una gran aceptación por parte del alumnado. Se ha detectado un grado de concienciación notable con los temas tratados, así como diversas inquietudes que revelan el cambio de actitud de la sociedad, y concretamente de la juventud, hacia una nueva realidad sostenible que ha venido para quedarse. Todo ello ha motivado al profesorado de estas asignaturas a contextualizar y profundizar en aquellos temas considerados de obligado conocimiento para los egresados en ADE.

En este artículo se pretende ir más allá e introducir al alumno en el aprendizaje y puesta en práctica de los ODS desde la impartición de materias básicas de sus estudios. Concretamente, se trata de complementar los estudios tradicionales con nociones de ODS cuya puesta en práctica a nivel empresarial permitirá enseñar a como reducir las desigualdades existentes y la creación de espacios verdes que, en definitiva, mejoren la calidad de vida de la población. Por tanto, se trata de crear concienciación ciudadana e ir forjando el camino para su intrusión en la esfera empresarial, donde todavía queda mucho trabajo por realizar.

\section{Referencias}

COMISIÓN DE COMERCIO Y DESARROLLO SOSTENIBLE (2017). Better business, better world. Disponible en: https://www.unglobalcompact.org/docs/news_events/9.3/betterbusiness- better-world.pdf

CRUE (2019a). El papel de la Universidad ante los Objetivos de Desarrollo Sostenible. http://www.crue.org/Comunicacion/Noticias/La\%20Universidad\%20en\%20los\%20Objetivos\%20 del\%20Desarrollo\%20Sostenible.aspx

CRUE (2019b). Memoria de Acciones Crue en materia de Agenda 2030. Disponible en: http://www.crue.org/Documentos\%20compartidos/Sectoriales/Sostenibilidad/2019.07.22\%20Me moria\%20Crue\%20ODS\%202018_vf.pdf

Medina, R (2019). Los objetivos de desarrollo sostenible como estrategia empresarial. EAE Business School. 
PROGRAMA DE LAS NACIONES UNIDAS PARA EL DESARROLLO - PNUD (2016). Objetivos de desarrollo sostenible. Disponible http://www.undp.org/content/undp/es/home/sustainabledevelopment-goals.html.

RED ESPAÑOLA DEL PACTO MUNDIAL (2016). El sector privado ante los ODS. Guía práctica para la acción. Disponible en: http://www.pactomundial.org/wpcontent/uploads/2016/09/Guia_ODS_online.pdf

RED ESPAÑOLA DEL PACTO MUNDIAL DE NACIONES UNIDAS. (2020). ODS-A O -4: Elliderazgo-empresarial.Disponible en: https://www.pactomundial.org/wpcontent/uploads/2020/01/ODS-A\%C3\%B1o-4.pdf

Remacha, M (2017). Empresa y objetivos de desarrollo sostenible. Cuadernos de la Catedra CaixaBank de Responsabilidad Social Corporativa. No 34. IESE. Business School.

SDSN (2017). Como empezar con los ODS en las universidades. Australia, New Zealand and Pacific Edition. Sustainable Development Solutions NetworK. 